\begin{tabular}{|c|l|}
\hline Title & Interplay of First-Order Kinetic and Thermodynamic Phase T ransitions in Heterogeneous Catalytic Reactions \\
\hline Author(s) & Zhdanov, V ladimir P.; Matsushima, Tatsuo \\
\hline Citation & $\begin{array}{l}\text { Physical Review Letters, 98(3), 036101 } \\
\text { https://doi.org/10.1103/PhysRevLet.98.036101 }\end{array}$ \\
\hline Issue Date & 2007-01-19 \\
\hline Doc URL & http://hdl.handle.net/2115/18845 \\
\hline Rights & Copyright $\odot 2007$ A merican Physical Society \\
\hline Type & article \\
\hline File Information & PRL98 3.pdf \\
\hline
\end{tabular}

Instructions for use 


\title{
Interplay of First-Order Kinetic and Thermodynamic Phase Transitions in Heterogeneous Catalytic Reactions
}

\author{
Vladimir P. Zhdanov ${ }^{1,2, *}$ and Tatsuo Matsushima ${ }^{1}$ \\ ${ }^{1}$ Catalysis Research Center, Hokkaido University, Sapporo 001-0021, Japan \\ ${ }^{2}$ Boreskov Institute of Catalysis, Russian Academy of Sciences, Novosibirsk 630090, Russia
}

(Received 13 September 2006; published 17 January 2007)

\begin{abstract}
Using the rate constants obtained on the basis of independent transient measurements and density functional theory calculations, we perform Monte Carlo (MC) simulations of the bistable kinetics of the $\mathrm{N}_{2} \mathrm{O}-\mathrm{CO}$ reaction on $\mathrm{Pd}(110)$ at $450 \mathrm{~K}$. In the absence of lateral interactions, the $\mathrm{MC}$ technique predicts a wide hysteresis loop in perfect agreement with the mean-field analysis. With attractive substrate-mediated lateral interactions resulting in the formation of $(1 \times 2) \mathrm{O}$ islands and reducing the reaction rate inside islands, the hysteresis is found to be dramatically (about 5 times) narrower. This finding explains why the first-order kinetic phase transition experimentally observed in this reaction is not accompanied by hysteresis.
\end{abstract}

PACS numbers: 68.35.Rh, 82.20.Wt, 82.40.Np

Because of their practical importance [1] and richness of behavior [2], the heterogeneous catalytic reactions (HCR) have long attracted attention of chemists, physicists, and mathematicians. Customarily, HCR are studied by varying governing parameters (e.g., reactant pressure). Since the early 1920s, such measurements were found to sometimes show a stepwise change in the reaction rate and adsorbate coverages (see Refs. [3-6] for a historical overview and recent studies, respectively). This feature, referred to as "a first-order kinetic phase transition" (KPT) (continuous KPT were predicted more recently [7]), is usually described by using the conventional mean-field (MF) kinetic equations, implying the adsorbed overlayer to be in the one-phase state, or MC simulations [4,8]. Mathematically, it represents a saddle-node bifurcation. If a governing parameter is varied back and forth, in agreement with the experiments, the corresponding MF models predict hysteresis. The available MC simulations [9] indicate that with decreasing lattice size, $L$, the hysteresis may disappear but usually it happens at fairly small lattices (for $L$ lower or about 20).

In real systems, the HCR kinetics are often influenced by adsorbate-adsorbate lateral interactions $[10,11]$. The effect of repulsive lateral interactions (such interactions result in order-disorder phase transitions) on first-order KPT has already been studied in detail $[4,12]$. The models with attractive lateral interactions have been analyzed as well (see [2(b),13]) but with the emphasis on the simplest reaction schemes, including molecular reactant adsorption and reaction, so that the stepwise changes in the kinetics are either lacking or occur due to lateral interactions. In this Letter, we scrutinize the likely effect of attractive lateral interactions on a true first-order KPT or, more specifically, show for a realistic reaction scheme how a KPT can be influenced by island formation. Physically, the island formation represents an example of phase separation and accordingly should be described in terms of the theory of first-order thermodynamic phase transitions. Thus, basically, our goal is to study the interplay of first-order kinetic and thermodynamic phase transitions.

To motivate our study in more detail, we refer to recent measurements [6] of the steady-state kinetics of the $\mathrm{N}_{2} \mathrm{O}-\mathrm{CO}$ reaction on $\mathrm{Pd}(110)$ at $P_{\mathrm{N}_{2} \mathrm{O}}=3.3 \times 10^{-6}$ Torr and $450 \leq T \leq 520 \mathrm{~K}$. With variation of $P_{\mathrm{CO}}$ between $4 \times 10^{-8}$ and $2.5 \times 10^{-6}$ Torr back and forth step by step (with the time interval of $360 \mathrm{~s}$ ) by a factor of about 1.5 (at $T=450 \mathrm{~K}$ ) or somewhat lower (at higher temperatures), this reaction is found to exhibit a first-order KPT at $T<500 \mathrm{~K}$. With decreasing temperature, the critical pressure becomes lower and, as expected, the corresponding ratio of the reaction rates of the high- and low-reactive regimes increases and reaches about 1 order of magnitude at $T=450 \mathrm{~K}$. Surprisingly, a hysteresis was, however, not observed even at this temperature. The corresponding MF kinetic model based on independent transient measurements and density-functional theory (DFT) calculations was demonstrated to predict a well-developed hysteresis. Although the MF model makes it possible to quantitatively describe the reaction kinetics assuming the KPT to occur along the equistability line, the reason why in reality the hysteresis is either lacking or much narrower compared to that predicted by using the MF approximation or expected on the basis of the experience accumulated in studies of other reactions, is still open for debate. Here, we present MC simulations showing that the likely explanation of this discrepancy is that the reaction is accompanied by a firstorder thermodynamic phase transition in the adsorbed overlayer or more specifically by the formation of oxygen $(1 \times 2)$ islands (the structure of such islands is shown below in Fig. 3).

The reaction under consideration includes $\mathrm{N}_{2} \mathrm{O}$ adsorption followed by rapid desorption or dissociation with subsequent $\mathrm{N}_{2}$ desorption. In fact, the latter three processes occur simultaneously with or just after adsorption. For this 
reason, all these processes can be replaced in simulations by a single lumped step,

$$
\left(\mathrm{N}_{2} \mathrm{O}\right)_{\text {gas }} \rightarrow\left(\mathrm{N}_{2}\right)_{\text {gas }}+\mathrm{O}_{\mathrm{ad}}
$$

where the subscripts "gas" and "ad" correspond to gasphase and adsorbed species, respectively. In addition, CO adsorbs reversibly and react with oxygen,

$$
\begin{gathered}
\mathrm{CO}_{\mathrm{gas}} \rightleftharpoons \mathrm{CO}_{\mathrm{ad}}, \\
\mathrm{CO}_{\mathrm{ad}}+\mathrm{O}_{\mathrm{ad}} \rightarrow\left(\mathrm{CO}_{2}\right)_{\mathrm{gas} .}
\end{gathered}
$$

The rate of step (1) rapidly decreases with increasing coverage. To be specific, we assume that this step occurs provided that $\mathrm{N}_{2} \mathrm{O}$ collides with a vacant site and the four nearest-neighbor (NN) sites are vacant. With these assumptions, the generic MF kinetic equations for the reactant coverages are as follows:

$$
\begin{gathered}
d \theta_{\mathrm{O}} / d t=k_{\mathrm{dis}} P_{\mathrm{N}_{2} \mathrm{O}}\left(1-\theta_{\mathrm{CO}}-\theta_{\mathrm{O}}\right)^{5}-k_{\mathrm{r}} \theta_{\mathrm{CO}} \theta_{\mathrm{O}}, \\
d \theta_{\mathrm{CO}} / d t=k_{\mathrm{ad}} P_{\mathrm{CO}}\left(1-\theta_{\mathrm{CO}}-\theta_{\mathrm{O}}\right)-k_{\mathrm{des}} \theta_{\mathrm{CO}} \\
-k_{r} \theta_{\mathrm{CO}} \theta_{\mathrm{O}},
\end{gathered}
$$

where $P_{\mathrm{N}_{2} \mathrm{O}}$ and $P_{\mathrm{CO}}$ are the reactant pressures, and $k_{\mathrm{dis}}$, $k_{\mathrm{ad}}, k_{\mathrm{des}}$, and $k_{r}$ are the rate constant of steps (1)-(3).

Typical steady-state MF reaction kinetics, calculated with the parameters $k_{\mathrm{dis}}=5.9 \times 10^{4} \mathrm{~s}^{-1} \mathrm{Torr}^{-1}, k_{\mathrm{ad}}=$ $4.1 \times 10^{5} \mathrm{~s}^{-1}$ Torr $^{-1}, k_{\mathrm{des}}=2.5 \times 10^{-2} \mathrm{~s}^{-1}$, and $k_{r}=$ $21 \mathrm{~s}^{-1}$ corresponding to $T=450 \mathrm{~K}$, are shown in Figs. 1 and 2 (top panels). For validation of these parameters, see Ref. [6]. Note that due to repulsive CO-CO lateral interactions the $\mathrm{CO}$ desorption rate constant $k_{\text {des }}$ depends on coverage [6]. In the context of our discussion, this effect is of minor importance and accordingly neglected in order to reduce the number of model parameter. The value of $k_{\text {des }}$ used here corresponds to $\theta_{\mathrm{CO}}=0.25$.

On $\mathrm{Pd}(110)$, the oxygen adsorption is well known to result in surface restructuring with the formation of $(1 \times 2)$ "missing-row" islands [14]. During the high reactive regime of the $\mathrm{N}_{2} \mathrm{O}-\mathrm{CO}$ reaction, when the surface is covered primarily by oxygen, the LEED patterns are also indicative of the formation of similar $(1 \times 2)$ or $(1 \times 3)$ islands. In this case, the full-scale MC simulations of the reaction kinetics should explicitly include the O-induced rearrangements of Pd atoms. Such simulations would be far from generic due to the multitude of parameters. To keep the model as simple as possible, we take the rearrangements of $\mathrm{Pd}$ atoms into account implicitly by introducing effective strongly anisotropic $\mathrm{O}-\mathrm{O}$ lateral interactions resulting in the formation of $(1 \times 2) \mathrm{O}$ islands (the term "effective" implies the Pd-mediated interactions). Specifically, adsorption is considered to occur on a $L \times L$ square lattice with periodic boundary conditions. The NN O-O lateral interactions along the $X$ and $Y$ axes (in Fig. 3 below, these axes are horizontal and vertical) are considered to be, respec-

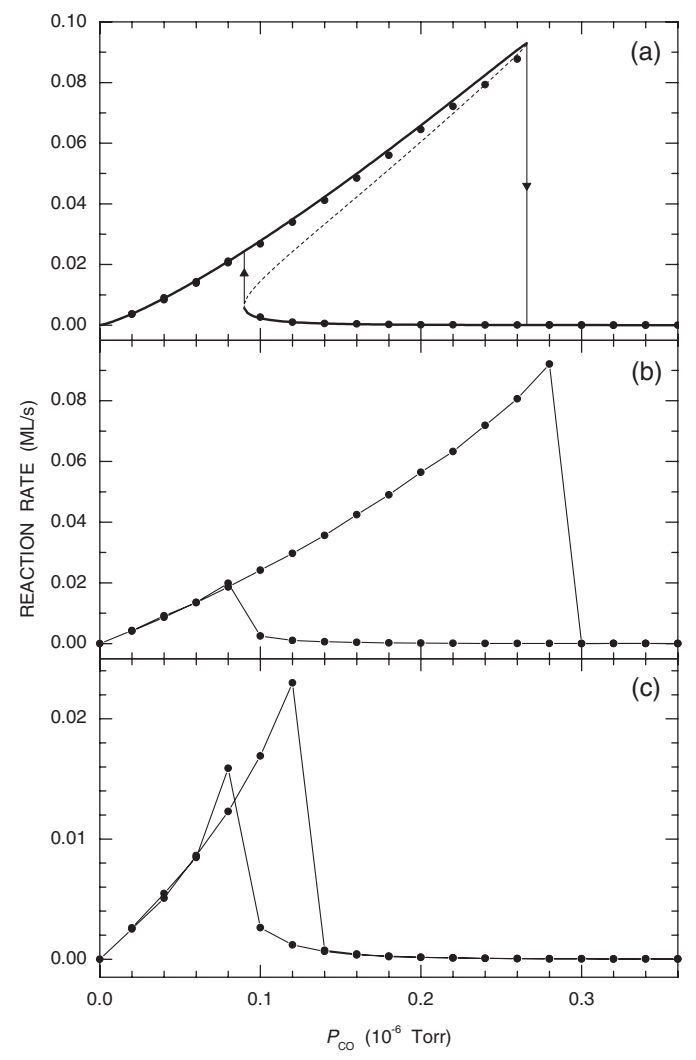

FIG. 1. Reaction rate as a function of $\mathrm{CO}$ pressure for $P_{\mathrm{N}_{2} \mathrm{O}}=$ $3.3 \times 10^{-6}$ Torr and $T=450 \mathrm{~K}$ : (a) The thick solid and thin dashed lines show, respectively, the stable and unstable MF regimes. The thin solid lines marked by arrows indicate the jumps corresponding to the hysteresis loop. The solid circles represent the $\mathrm{MC}$ kinetics in the absence of lateral interactions. (b) MC kinetics influenced by lateral interactions with $\alpha=0$. (c) As (b) for $\alpha=0.5$. The average statistical error in the MC results is lower than or comparable to the size of the data points.

tively, repulsive and attractive, $\epsilon_{\mathrm{nn}}^{x}=3 k_{B} T$ and $\epsilon_{\mathrm{nn}}^{y}=$ $-3 k_{B} T$. The next-NN O-O lateral interactions along the $X$ axis is assumed to be attractive, $\epsilon_{\mathrm{nnn}}^{x}=-3 k_{B} T$. To reduce the number of model parameters, the absolute values of the interactions introduced are chosen to be equal. The other lateral interactions are neglected.

The $\mathrm{O}-\mathrm{O}$ lateral interactions influence $\mathrm{O}$ diffusion and reaction (3) between $\mathrm{O}$ and $\mathrm{CO}$. We consider that the $\mathrm{O}$ and $\mathrm{CO}$ diffusion occurs via jumps to $\mathrm{NN}$ vacant site and employ the conventional Metropolis rule for the normalized probability of $\mathrm{O}$ jumps $\left(P_{\mathrm{dif}}=1\right.$ for $\epsilon_{f} \leq \epsilon_{i}$, and $P_{\mathrm{dif}}=\exp \left[-\left(\epsilon_{f}-\epsilon_{i}\right) / k_{B} T\right]$ for $\epsilon_{f}>\epsilon_{i}$, where $\epsilon_{i}$ and $\epsilon_{f}$ are the energies of an $\mathrm{O}$ atom in the initial and final states, respectively). The use of the Metropolis dynamics is physically reasonable because the $\mathrm{O}-\mathrm{O}$ lateral interaction is predominantly attractive. Note also that the kinetics of the island growth is rather insensitive to the detail of the diffusion dynamics.

For the normalized probability of reaction between NN $\mathrm{O}$ and $\mathrm{CO}$, we use $P_{r}=1$ for $\epsilon_{i}>0$, and $\exp \left(\alpha \epsilon_{i} / k_{B} T\right)$ 

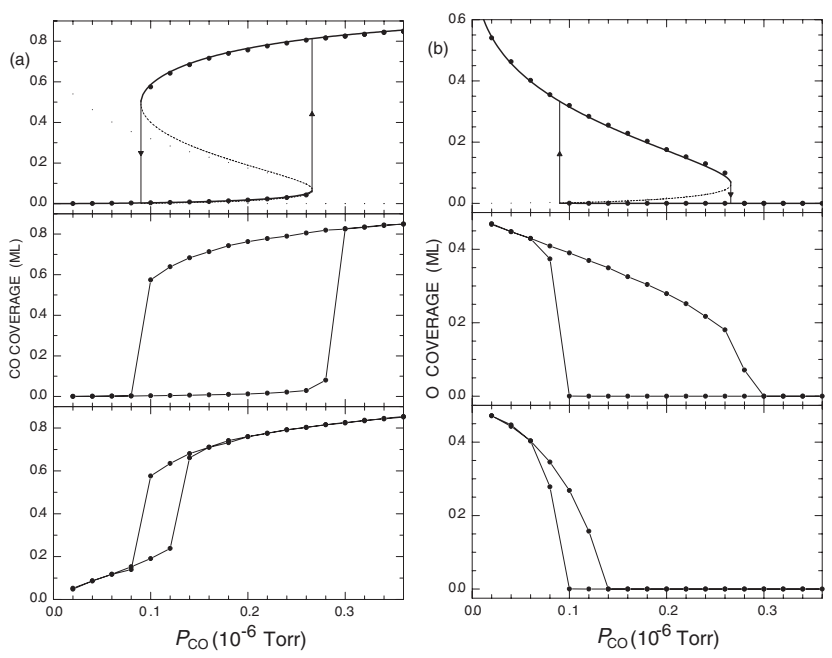

FIG. 2. (a),(b): $\mathrm{CO}$ and $\mathrm{O}$ coverages corresponding to the kinetics shown in Fig. 1.

for $\epsilon_{i}<0$, where $\alpha(0 \leq \alpha \leq 1)$ is the parameter taking into account that the effect of the lateral interaction in the initial state on the reaction rate can be reduced due to the lateral interaction in the activated state [11]. With $\alpha=0$, this rule predicts that the reaction probability is independent of the occupation of adjacent sites. If $\alpha>0$ (this is expected to correspond to reality), the reaction probability becomes lower in the situations (e.g., inside islands) when the reacting $\mathrm{O}$ atom has $\mathrm{O}$ neighbors in $\mathrm{NN}$ sites.

In $\mathrm{MC}$ simulations, the dimensionless probabilities of elementary events are obtained by normalizing the corresponding rate constants to the properly defined maximum rate constant $k_{\max }$. In the reaction under consideration, the fastest process is $\mathrm{CO}$ diffusion, and accordingly we use $k_{\max }=k_{\mathrm{dif}}^{\mathrm{CO}}+k_{r}+k_{\mathrm{des}}$, where $k_{\mathrm{dif}}^{\mathrm{CO}}$ is the CO jump rate constant in the case when all the NN sites are vacant, and $k_{r}$ is the maximum reaction rate, i.e., the reaction rate constant corresponding to the configurations with $\epsilon_{i}=0$. [Note that the rate constants, used here and below for the steps forming the catalytic cycle, are identical to those employed in Eqs. (4) and (5).]

With the specification above, the MC runs consist of sequential trials to realize elementary events on a randomly chosen site. (i) If the site selected is vacant, the $\mathrm{CO}$ adsorption or $\mathrm{N}_{2} \mathrm{O}$ dissociative adsorption are performed with probabilities $k_{\mathrm{ad}} P_{\mathrm{CO}} / k_{\max }$ and $k_{\mathrm{dis}} P_{\mathrm{N}_{2} \mathrm{O}} / k_{\max }$, respectively. The $\mathrm{N}_{2} \mathrm{O}$ dissociation is realized provided that all the NN sites are vacant. (ii) If the site chosen is occupied by $\mathrm{CO}$, the $\mathrm{CO}$ desorption, reaction, or diffusion-jump events are executed with probabilities $k_{\mathrm{des}} / k_{\max }, k_{r} / k_{\max }$, and $k_{\text {dif }}^{\mathrm{CO}} / k_{\max }$. If the selected event is reaction, one of the NN sites is chosen at random and the event is realized provided that the latter site is occupied by $\mathrm{O}$ and $\rho \leq P_{r}$, where $\rho$ $(0 \leq \rho \leq 1)$ is a random number (this reduction of the reaction probability takes into account the effect of the O$\mathrm{O}$ lateral interactions on the reaction rate). If the selected event is diffusion, one of the $\mathrm{NN}$ sites is chosen at random and the event is realized provided that the latter site is vacant. (iii) If the site chosen is occupied by $\mathrm{O}$, one of the $\mathrm{NN}$ site is selected at random and if the latter site is vacant the $\mathrm{O}$ diffusion-jump event is executed with probability $P_{\text {dif }} k_{\text {dif }}^{\mathrm{O}} / k_{\max }$, where $k_{\text {dif }}^{\mathrm{O}}$ is the $\mathrm{O}$ jump rate constant in the case when all the adjacent sites are vacant. [Note that there is no need to perform a reaction trial on this stage, because it is executed on stage (ii).] (iv) After each MC trial, the time is incremented by $\Delta t=\left|\ln \left(\rho^{\prime}\right)\right| /\left(L^{2} k_{\max }\right)$, where $\rho^{\prime}$ is another random number.

To compare with the experiment [6] and MF calculations described, the MC simulations have been performed at $T=450 \mathrm{~K}$ with the same values of the adsorption, desorption, and reaction rate constants as in the MF case. In reality, at this temperature, $\mathrm{CO}$ diffusion is about 7 orders of magnitude faster than reaction (3). In our simulations, $\mathrm{CO}$ diffusion was 3 orders of magnitude faster than reaction [specifically, we used $\left(k_{\mathrm{des}}+k_{r}\right) / k_{\max }=10^{-3}$ ]. Further increase (or decrease by 1 order of magnitude) of the rate of $\mathrm{CO}$ diffusion was proved to not influence the results. For $\mathrm{O}$ diffusion, we employed $k_{\text {dif }}^{\mathrm{O}} / k_{\max }=10^{-1}$; i.e., this process was 2 orders of magnitude faster than reaction (3). The $\mathrm{CO}$ pressure was increased and then decreased step by step by $2 \times 10^{-8}$ Torr. The time interval of $100 \mathrm{~s}$ between the steps was chosen to be somewhat shorter than in the experiment in order to compensate a smaller value on the pressure increment used in the simulations. The data points were obtained during the last $10 \mathrm{~s}$ of each step, using a lattice with $L=200$. The increase or decrease of $L$ by a factor of 2 were proved to not influence the results.

First, we have performed MC simulations in the absence of lateral interactions. In this case, all the ingredients (except fluctuations) of the MC model are the same as in the MF model, and the MC and MF results [Figs. 1 and 2 (top panels)] are found to be in perfect agreement. The important point is that the MF model ignores fluctuations and accordingly predicts the upper width of a hysteresis. The observed agreement between the MC and MF results indicates that for the reaction under consideration the MC technique predicts the upper hysteresis width as well.

Second, we have executed MC simulations [Figs. 1 and 2 (middle panels)] with lateral interactions for $\alpha=0$. In this case, the lateral interactions result in the formation of $(1 \times 2)$ O islands [Fig. 3(a)] but do not influence the reaction rate of NN reactant pairs. Despite the island formation, the hysteresis is found to be nearly the same as in the MF case [cf. Figs. 1(a) and 1(b)].

Third, we have scrutinized the situation (with $\alpha=0.5$ ) when the lateral interactions result in the formation of $(1 \times$ 2) $\mathrm{O}$ islands [Fig. 3(b)] and reduce the reaction rate of $\mathrm{NN}$ reactant pairs inside the islands. In this case, the hysteresis is found [Figs. 1 and 2 (bottom panels)] to be dramatically (about 5 times) narrower than in the MF case. In particular, 
(a)

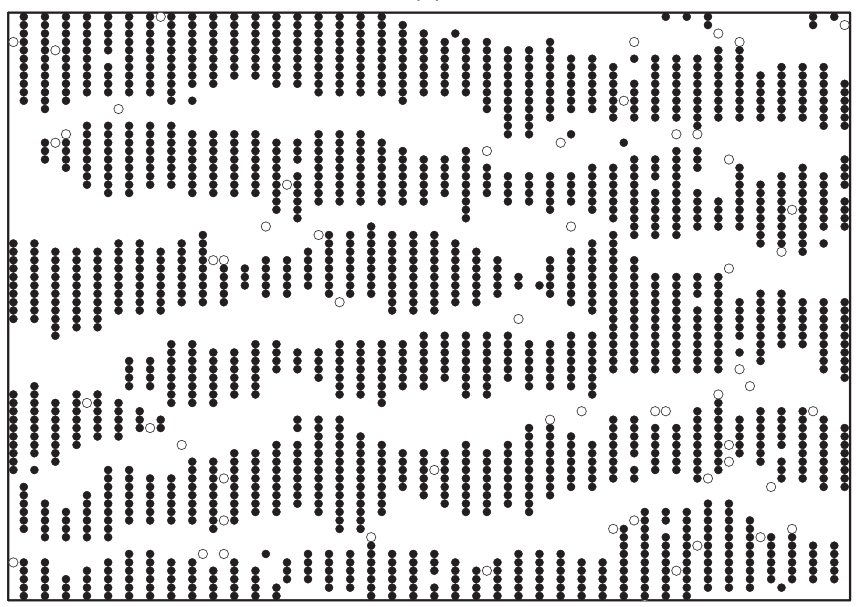

(b)

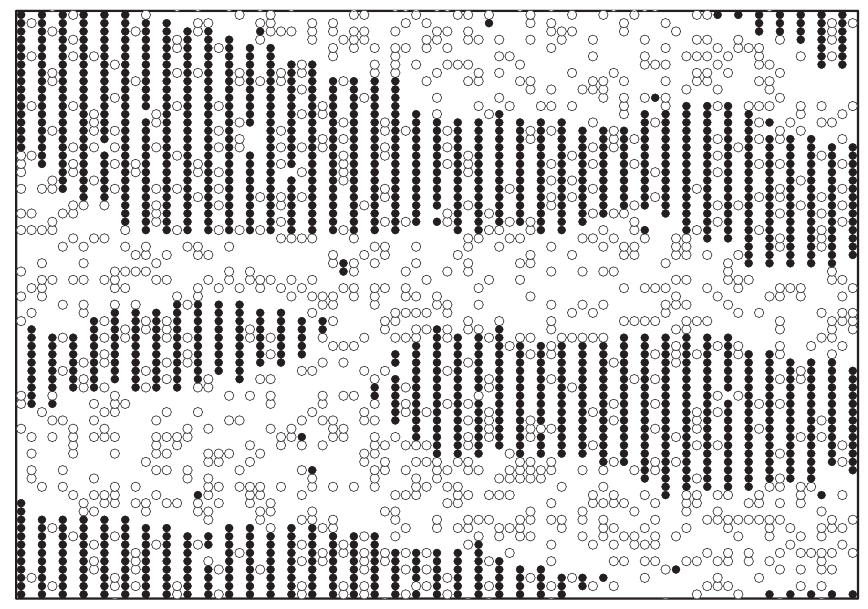

FIG. 3. Snapshots of a $80 \times 70$ fragment of the $200 \times 200$ lattice during the high reactive regime: (a) at $P_{\mathrm{CO}}=$ $1.8 \times 10^{-7}$ Torr for the kinetics exhibited in Fig. 1(b); (b) at $P_{\mathrm{CO}}=10^{-7}$ Torr for the kinetics presented in Fig. 1(c). The solid and open circles indicate $\mathrm{O}$ and $\mathrm{CO}$, respectively. The vacant sites are not shown. Note that oxygen atoms form anisotropic $(1 \times 2)$ islands (in reality the formation of such islands is accompanied by rearrangement of surface Pd atoms).

although the amplitude of the hysteresis is appreciable (close to that observed in the experiment [6]), the ratio of the upper and lower critical CO pressures is only about 1.3; i.e., the hysteresis is narrower than the distance between the data points in the experiment [6]. This seems to be the likely reason why the hysteresis was not observed in the experiment.

In summary, we have performed $\mathrm{MC}$ simulations of the bistable kinetics of the $\mathrm{N}_{2} \mathrm{O}-\mathrm{CO}$ reaction on $\mathrm{Pd}(110)$ by using the well-established reaction mechanism with the rate constants, obtained on the basis of independent transient measurements and DFT calculations, and taking into account the experimentally observed substrate-mediated formation of $(1 \times 2) \mathrm{O}$ islands. For the key reaction step (3), we have employed three sets of lateral interactions making it possible to describe various physically reasonable situations. In the reference case (without lateral interactions and island formation), the MC technique and MF calculations predict a wide hysteresis loop. With the island formation, the width of a hysteresis loop is found to be appreciably narrower provided that the lateral interactions reduce the reaction rate inside islands. This finding helps to rationalize the experimental data obtained for this reaction [6] and is also of interest in a more general context. In particular, the physics behind the bistability in the $\mathrm{N}_{2} \mathrm{O}-\mathrm{CO}$ reaction on $\mathrm{Pd}$ is similar to that in $\mathrm{CO}$ or $\mathrm{H}_{2}$ oxidation on Pt-group metals [4], and accordingly our results may conceptually be useful for the latter reactions as well.

*Electronic address: zhdanov@catalysis.ru

[1] J. M. Thomas and W. J. Thomas, Principles and Practice of Heterogeneous Catalysis (VCH, Veinheim, 1997).

[2] (a) F. Schüth et al., Adv. Catal. 39, 51 (1993); R. Imbihl et al., Chem. Rev. 95, 697 (1995); G. Ertl, Adv. Catal. 45, 1 (2000); (b) V. P. Zhdanov, Surf. Sci. Rep. 45, 231 (2002).

[3] V.I. Bykov et al., Kinetic Models of Catalytic Reactions (Elsevier, Amsterdam, 1991).

[4] V.P. Zhdanov and B. Kasemo, Surf. Sci. Rep. 20, 111 (1994).

[5] M. Berdau et al., J. Chem. Phys. 110, 11551 (1999); B. L. M. Hendriksen et al., Phys. Rev. Lett. 89, 046101 (2002); S. Wehner et al., Chem. Phys. Lett. 370, 126 (2003); V. Johanek et al., Science 304, 1639 (2004).

[6] V.P. Zhdanov, Y. Ma, and T. Matsushima, Surf. Sci. 583, 36 (2005).

[7] R. M. Ziff et al., Phys. Rev. Lett. 56, 2553 (1986).

[8] D. J. Liu and J. W. Evans, Multiscale Model Simul. 4, 424 (2005); E. V. Albano, Heter. Chem. Rev. 3, 389 (1996).

[9] V.P. Zhdanov et al., Surf. Sci. 496, 251 (2002); N. Pavlenko et al., Phys. Rev. E 68, 016212 (2003).

[10] H.C. Kang and W.H. Weinberg, Chem. Rev. 95, 667 (1995).

[11] V.P. Zhdanov, Elementary Physicochemical Processes on Solid Surfaces (Plenum, New York, 1991).

[12] D. J. Liu and J. W. Evans, J. Chem. Phys. 124, 154705 (2006).

[13] D. G. Vlachos et al., Surf. Sci. 249, 248 (1991); V.P. Zhdanov, Phys. Chem. Chem. Phys. 7, 2399 (2005); M. G. Clerc et al., Phys. Rev. Lett. 97, 176102 (2006).

[14] R. A. Bennett et al., Surf. Sci. 401, 72 (1998). 